\title{
Passing sequences towards field goals and penalty corners in men's field hockey
}

\author{
MOHD ARIFF $\triangle$, SULAIMAN NORASRUDIN, ADNAN RAHMAT, ISMADI SHARIMAN \\ Performance Analysis Lab, Faculty of Sports Science and Recreation, Universiti Teknologi MARA (UiTM), Shah \\ Alam, Selangor
}

\begin{abstract}
Ariff, M., Norasrudin, S., Rahmat, A., \& Shariman, I. (2015). Passing sequences towards field goals and penalty corners in men's field hockey. J. Hum. Sport Exerc., 10(Proc2), pp.S638-S647. Modern field hockey relies on effective game play within wellstructured ball movement as its multidimensional factors in contributing field goals and penalty corners. Therefore, the objective of the study was to analyse the effect of passing sequences (longer passing sequence and shorter passing sequence) towards field goals and penalty corners in men's field hockey. Passing sequence referred to how many touches or passing made towards obtaining field goals or penalty corners won by the attacking team. Longer passing sequence was passing made more than five times, meanwhile shorter passing sequence was passing made less than four times. Analysis of 24 footages/ video taken from World Hockey League Semi Finals at Johor Bahru, 2013, including 8 countries (Germany, Argentina, England, Korea, Malaysia, Japan, Pakistan, and South Africa) competing to qualify for World Cup 2014. The analytical process focused on two different passing sequences in attacking on field goals and penalty corners in men's field hockey. Two reliability tests, (intra and inter observer test) were conducted on researcher before going through the analysis process. All the footage/video were analysed by using computerized analysis software (SPORTCODE ELITE). A Wilcoxon Signed Ranked Test was deemed the appropriate statistical method to determine the effect of this study. Results showed that there were significant different between shorter passing and longer passing sequence towards field goals when, $(z=-4.05, p<.05)$, and the median for shorter passing sequences is greater than that of the longer passing sequences towards field goals. Meanwhile, for penalty corners, the data also showed that shorter passing sequences were more effective and beneficial in men's field hockey when $(z=-4.29, p<.05)$, and the median for shorter passing sequences is greater than that of the longer passing sequences towards penalty corners. Moreover other findings from this study also support that shorter passing sequence is more effective during attacking in men's field hockey when shown that $\mathrm{D}$ penetration more often occurred compare to longer passing sequence $(z=-4.28, p<.05)$. It is important for all team to use an effective tactical play during attacking task especially for sports which emphasize on striking invasion like field hockey. Furthermore, in this modern field hockey, less sequence of passing suit with quick movement to obtain field goals or penalty corners before the opponent reorganize their defend. These findings could be used to support any level of field hockey coaches to implement during training as to improve and enhance their team attacking ability during competition. Further research could to be conducted on the correlation between passing sequence and basics skills performance in field hockey. Key words: PASSING SEQUENCE, FIELD GOALS, PENALTY CORNERS, D PENETRATION.
\end{abstract}

Corresponding author. Performance Analysis Lab, Faculty of Sports Science and Recreation, Universiti Teknologi MARA (UiTM), Shah Alam, Selangor

E-mail: arif@uniten.edu.my

Asia Pacific Conference on Performance Analysis of Sport, 21-24 April 2014. Langkawi, Malaysia.

JOURNAL OF HUMAN SPORT \& EXERCISE ISSN 1988-5202

(c) Faculty of Education. University of Alicante

doi:10.14198/jhse.2015.10.Proc2.01 


\section{INTRODUCTION}

Performance analysis in sports nowadays gives meaningful benefits especially in enhancing team or athlete's performance. Its role work as a guideline for coaching staff to do some adjustment on athlete's technical execution and also team's tactical implementation. Parallel with Hughes and Bartlett (2002), performance analysis scope are focuses on helping in improving technically, tactically and also movement pattern of both athlete and team. The most initial method in performance analysis is notational, and currently this method advance with combination of technology to ensure the quality of the analysis or feedback valid and reliable.

The priority of sports striking invasion like field hockey are scoring more goal and avoid concede more away goal to become a winner at the end of any matches. In field hockey, the $D$ area becomes a specific area to make an attempt on goal and to score the goal. On the other hand, any infringement of rules by the defender inside 25 meter and the $D$ area could give an advantage to the attacking side to score by field goal or conversion of the penalty corner. Previous study in distinguish playing pattern between winning and losing team in female field hockey indicate winning team were the team that made most penetration on opponent's D area (Sofwan et al., 2012). Meanwhile, other study on football indicate there are more goals scored inside the score box possession, rather than outside box, because more accurate shots on target have increase chances of scoring goals (Tenga et al., 2010).

The keys to penetrate that area are each team needs to emphasize and precise on tactical and technical aspects. One of the basic things in any sports striking invasion is passing. Passing can derive team to score the goal, eliminate the opponent by delivered the ball forward well, and clear any opponent threat from danger zone. In field hockey, other part of attacking objective is to obtain penalty corner.

Furthermore, passing is also part of tactical implementation during launching an attack. Previous study made in football stated two different tactical approaches applied on passing which are longer passing sequence (possession approach) and shorter passing sequence (direct play approach) (Armatas, 2007; Castellano, 2012; Hughes \& Franks, 2005; Tenga, et al., 2010; Yiannakos \& Armatas 2006). Longer passing sequence is sequence more than five passing, meanwhile shorter passing sequence is sequence less than four passing. This sequence of passing previously reported as the determinant for each team to score a goal.

Football and field hockey are interrelated due to the similarity of the outcome based even though quietly different in rules. Previously reported that shorter passing sequence in football lead to more goal being scored (Reep \& Benjamin, 1968), conversely, other previous study indicate that longer passing sequence also lead to goal being scored in football (Castellano, 2012; Hughes \& Franks, 2005; Tenga et al., 2010; Yiannakos \& Armatas, 2006).

Even though both of the findings look contradict, but due to certain external factors, for instance different coaching approach influenced attacking style that contributed as the findings of those study. As limited study conducted on passing sequence towards goals being scored in field hockey, therefore this conducted study wants to analyze various passing sequence (longer or shorter passing sequence) towards field goal and penalty corner in hockey. 


\section{MATERIAL AND METHODS}

\section{Footage/Tournament Selection}

The footage/video were taken from the World Hockey League Semi Final Johor Bahru 2013 (N = 24). There were 8 country competed in that tournament (Germany, Argentina, England, Korea, Malaysia, Japan, Pakistan, and South Africa). This tournament was selected due to as the platform to be qualified in the 2014 World Cup and all those teams compete were well prepared and showed highly quality of performance for each match.

\section{Procedures}

In order to measure the validity of researcher in collecting the data, current research has admin intraobserver and inter-observer reliability measurement. Intra observer test needs researcher to complete two different analyses on the same match and the interval between tests is one week. While for inter-observer, researcher have to complete one analysis on one selected match and the results were compared with the data from accredited analyst. Furthermore, current research also measures the total error of the analysis. Based on the measurement, intra-observer $(r=0.89)$, inter observer $(r=0.82)$ and total error measurement (TEM) $(4.8 \%)$ shows the acceptable values. All the footage was analyzed by analysis software (SPORTCODE ELITE).

\section{Variables and categories}

\section{Starting zone}

This study adopt the method on counting the sequence of passing from the previous study of offensive match play in professional football by (Tenga et al., 2012), the starting zone start when the players made a passing over the middle third (50 meter and above) until reach the back/goal line of the defender area.

\section{Types of passing sequence}

Longer passing sequence (possession play)

This sequence of passing made more than 5 times until the attacking team obtain the field goals or penalty corners.

Shorter passing sequence (direct play)

Sequence of passing made less than 4 times until the attacking team obtain the field goals or penalty corners.

\section{Passing sequence outcome}

\section{Field goals}

Scored by the attacking teams in the $\mathrm{D}$ area or the ball deflected by any of the defender body or equipment (stick or keeper's padding) and the ball legally enter the goal and declared goal by the umpire.

\section{Penalty corners}

The attacking teams obtain the advantage after the infringement of any rules by the defender in 25 meter area or inside the $\mathrm{D}$ area and declared foul by the umpire. 


\section{Other variables}

i. $\quad$ Passing sequence on $D$ penetration

ii. Ways of $D$ penetration

iii. Types of passing

iv. Ways of passing

v. Shot on goals

vi. Ways of field goals scored

vii. Area of field goals scored

viii. Penalty corners goals

ix. Penalty stroke goals

Statistical analysis

All the data were analyzed by using Wilcoxon Rank Test method to determine the effect of passing sequence towards field goals and penalty corners. Statistical evaluation made by using statistical software SPSS version 19.0, and the alpha value for significant of this test was set at $p<.05$.

\section{RESULTS}

\section{Descriptive analysis}

Descriptive analysis for this study as shown in table 1 indicate means, standard deviation, minimum frequency, maximum frequency, and sum of overall data included in this study.

Table 1. Descriptive analysis

\begin{tabular}{lcccc}
\hline & Mean & Min & Max & Sum \\
\hline LPS field goal & $.29 \pm .55$ & 0 & 2 & 7 \\
SPS field goal & $2.50 \pm 1.78$ & 0 & 6 & 60 \\
LPS penalty corner & $.13 \pm .45$ & 0 & 2 & 3 \\
SPS penalty corner & $5.75 \pm 2.52$ & 1 & 11 & 138 \\
LPS penalty stroke & - & - & - & - \\
SPS penalty stroke & $.17 \pm .48$ & 0 & 2 & 4 \\
& & & 4 & 21 \\
LPS penetration & $.88 \pm 1.08$ & 0 & 36 & 589 \\
SPS penetration & $24.54 \pm 6.04$ & 12 & & \\
& & & 13 & 209 \\
Ways of D penetration & & & 6 & 42 \\
push & $8.71 \pm 2.89$ & 3 & 4 & 29 \\
slap & $1.79 \pm 1.45$ & 0 & 3 & 6 \\
hit & $1.88 \pm 1.42$ & 0 & 2 & 14 \\
reverse & $1.17 \pm 1.24$ & 0 & 20 & 265 \\
flick & $.25 \pm .68$ & 0 & 10 & 67 \\
deflected & $.58 \pm .71$ & 0 & 7 & 4 \\
individual run & $11.46 \pm 4.75$ & 1 & 2 & \\
Penetration penalty corner & $4.58 \pm 2.04$ & 1 & & 5102 \\
Penetration field goals & $2.79 \pm 2.0$ & 0 & 293 \\
Penetration penalty stroke & $.17 \pm .48$ & 0 & 29 & \\
Types of passing & & & & \\
Short passing & $170.92 \pm 46.42$ & 92 & & \\
Long passing & $21.33 \pm 3.76$ & 15 & & \\
Ways of short passing & & & & \\
\hline
\end{tabular}




\begin{tabular}{|c|c|c|c|c|}
\hline push & $134.79 \pm 33.75$ & 78 & 203 & 3235 \\
\hline slap & $23.08 \pm 15.01$ & 7 & 77 & 554 \\
\hline hit & $1.83 \pm 1.63$ & 0 & 5 & 44 \\
\hline reverse & $8.71 \pm 4.24$ & 2 & 19 & 209 \\
\hline flick & $1.0 \pm 1.64$ & 0 & 6 & 24 \\
\hline deflected & $1.5 \pm 1.41$ & 0 & 5 & 36 \\
\hline \multicolumn{5}{|l|}{ Ways of long passing } \\
\hline push & $5.25 \pm 1.77$ & 3 & 9 & 126 \\
\hline slap & $9.21 \pm 4.68$ & 4 & 15 & 221 \\
\hline hit & $1.63 \pm 1.31$ & 0 & 4 & 39 \\
\hline reverse & $.29 \pm .55$ & 0 & 2 & 7 \\
\hline flick & $4.88 \pm 2.45$ & 0 & 10 & 117 \\
\hline deflected & $.08 \pm .28$ & 0 & 1 & 2 \\
\hline Field goals & $2.79 \pm 2.0$ & 0 & 7 & 67 \\
\hline Penalty corners & $7.33 \pm 3.51$ & 1 & 15 & 176 \\
\hline Penalty corners goals & $2.17 \pm 1.20$ & 0 & 6 & 52 \\
\hline Penalty stroke & $.21 \pm .51$ & 0 & 2 & 5 \\
\hline Penalty stroke goals & $.13 \pm .34$ & 0 & 1 & 3 \\
\hline Overall goals & $5.08 \pm 2.23$ & 2 & 10 & 122 \\
\hline \multicolumn{5}{|l|}{ Shot on goal } \\
\hline LPS shot on goal & $.33 \pm .57$ & & 2 & 8 \\
\hline SPS shot on goal & $8.88 \pm 1.62$ & 6 & 12 & 213 \\
\hline \multicolumn{5}{|c|}{ Ways of field goals scored } \\
\hline push & $.67 \pm .76$ & 0 & 2 & 16 \\
\hline slap & $.08 \pm .28$ & 0 & 1 & 2 \\
\hline hit & $.54 \pm .83$ & 0 & 3 & 13 \\
\hline reverse & $.58 \pm .83$ & 0 & 3 & 14 \\
\hline flick & - & - & - & - \\
\hline deflected & $.88 \pm .99$ & 0 & 3 & 22 \\
\hline \multicolumn{5}{|c|}{ Area of field goals scored } \\
\hline Right & $1.02 \pm .78$ & 0 & 2 & 24 \\
\hline Center & $.92 \pm .93$ & 0 & 3 & 22 \\
\hline Left & $.88 \pm .90$ & 0 & 3 & 21 \\
\hline
\end{tabular}

From the descriptive analysis in table 1 above, indicate most ways of $D$ penetration used in that tournament was individual run when 265 times recorded followed by push 209 times and the least ways to penetrate the $D$ were flick and deflected. Furthermore, overall penetration made were 610 times and from that figures its contributed for 67 field goals, 110 penalty corners rewarded, and 5 penalty stroke given.

There were 4102 short passing and only 512 long passing recorded for that tournament. The most ways for short passing was push when 3235 times recorded and least ways was flick only 24 times. Meanwhile for long passing the most ways used was slap when 221 recorded and the least ways used was deflected for only 2 times.

Overall goals recorded for this tournament were 122 goals, 67 contributed from field goals, on the other hand 176 penalty corners rewarded but only 52 goals succeed to score by overall team, meanwhile for penalty stroke there were 5 times rewarded and only 3 goals succeed as scoring. The most field goals 
scored by deflected when 22 times recorded and least ways used was slap only 2 times. Meanwhile the most area of field goals scored was on the right side followed by center and left side. In that tournament recorded 221 times of shot on goals made by all competing teams, 213 were from shorter passing sequence and only 8 times resultants from longer passing sequence.

Comparison on passing sequence

Table 2. indicate significant different between shorter passing sequence with longer passing sequence towards field goals, penalty corners, and D penetration

\begin{tabular}{cccc}
\hline & $\begin{array}{c}\text { SPS field goals - } \\
\text { LPS field goals }\end{array}$ & SPS penalty corners - LPS penalty corners & $\begin{array}{c}\text { SPS penetration - LPS } \\
\text { penetration }\end{array}$ \\
\hline Z & -4.05 & -4.29 & -4.29 \\
Sig $(2$ tailed $)$ & $.00^{*}$ & $.00^{*}$ & $.00^{*}$ \\
\hline
\end{tabular}

Table 3. showed mean, standard deviation and median for longer and shorter passing sequence on field goals, penalty corners, and $\mathrm{D}$ penetration

\begin{tabular}{ccc}
\hline & Mean & Median \\
\hline Longer passing sequence field goals & $.29 \pm .55$ & .00 \\
Shorter passing sequence field goals & $2.50 \pm 1.79$ & 2.00 \\
Longer passing sequence penalty corners & $.13 \pm .45$ & .00 \\
Shorter passing sequence penalty corners & $5.75 \pm 2.52$ & 6.00 \\
Longer passing sequence penetration & $.88 \pm 1.08$ & 1.00 \\
Shorter passing sequence penetration & $24.54 \pm 6.04$ & 24.00 \\
\hline
\end{tabular}

There were significant different between shorter passing sequence with longer passing sequence towards field goals $(z=-4.05, p=.00, p<.05)$ and penalty corners $(z=-4.29, p=.00, p<.05)$. Furthermore, there was significant different on shorter passing sequence with longer passing sequence on $D$ penetration when $(z=-4.28, p=.00, p<.05)$, as results shown in table 2 above. These results indicate shorter passing sequence was more effective and benefits towards obtaining field goals and penalty corners and it support by the median value of shorter passing sequence was greater than longer passing sequence as indicate in table 3 above.

\section{DISCUSSION AND CONCLUSION}

The finding of this study was significantly proved on the effect of different passing sequence (longer passing sequence and shorter passing sequence) towards field goals and penalty corners in men's field hockey. The statistical analysis showed that shorter passing sequence is more effective and beneficial during attacking in field hockey compare to longer passing sequence. The results was similar with some previous study that differentiate winning team in football when shorter passing sequence contributed more to obtained goals compare to longer passing sequence (Castellano, 2012; Hughes \& Franks, 2005; Reep \& Benjamin, 1968; Tenga et al., 2010; Yiannakos \& Armatas, 2006). Although these two sports were different, but both aimed were similar by netting more goals as possible to become a winner at the end of the match. 
Previous study by Lago and Martin (2007), reported that losing teams hold the ball more frequent compared to winning team in order to equalize the number of goal while prevent opposing team added more goals. Obviously winning team score more goals than losing team and some previous study made on effective offensive play in professional football indicate that winning teams tend to applied longer passing sequence during attacking (Hughes \& Franks, 2005; Reep \& Benjamin, 1968; Tenga et al., 2010; Yiannakos \& Armatas, 2006). The main reason for it is because this sequence of passing can ensure them to retain the ball longer and can make the defender situation imbalance thus exposed them on the tendency for mistakes due to eagerness to find the equalizer, while take the advantages to obtain more goals. On the other hand most of top football team in the world tends to keep the ball more longer compare to regular team or lower league team (Yiannakos \& Armatas, 2006). This explains how top teams can easily control the match with highly technical ability possessed by their players while lower league teams seem to struggle with the lack of this aspect.

For some reason in context of football, there is an offside terms where this rules disable the attacker to send the ball directly to the forwards. In order to break the offside line the attacker need an effective tactical to penetrate the defender area. On certain time they need to keep the ball for some time until their plan succeeds to break down the defender line. Conversely in field hockey, there is no offside rules which allow the attacking team to deliver the ball directly towards their forwards to make an attempt on scoring field goals or press defender for fouls in order to obtain the penalty corners. In addition, modern field hockey nowadays involve quick movement and the rules alterations by International Hockey Federation (FIH) that allowed any free hit of the out ball or any fouls to quickly taken make the game faster. These multidimensional factors could be the reasons or explanation of shorter passing sequence was more effective and beneficial during attacking in field hockey.

Besides alterations of the rules that make a ball movement more quick, the contribution of short sequence of passes and precision on delivering the ball made it easy for any forward or strikers to score goals, which have been proved when the most ways used in scoring was by deflected for about 22 times compared to other ways such as pushing and hitting. In other words field goals easily obtained as ball touched and directed upon the goal post.

As recorded in that tournament, the most area of field goals was scored is from the right side when recorded for 24 times, compared to center and left side. According to Sofwan, Norasrudin, Redzuan, and Mubin (2012), similar result showed for area of field goals scored and its looked like this position was the easiest ways to obtain the goals, mainly because the receiver collected the ball within their strong side position (forehand), thus faced directly towards the ball being delivered and more comfortable to execute deflection, hitting or pushing the ball towards the goal post. Converse result indicates by Amjad, Hussain, and Asadullah (2013), when recorded more direct goals or field goals origin from left side.

Recently FIH also altered one rules that give more advantages to the attacking team which was 'own goals'. In football, this term is very familiar when the opposite team's goalkeeper or the defender scored unintentionally to their own net. Believed the terms is still new in field hockey but nowadays more tactical are illustrated in order to exploit the weaknesses of the defender by often to hit the ball inside the D area with hope it struck the defender stick or touched the padding of goal keeper to make an attempt and count as a legal score. 
Football and field hockey are quite similar, but certain rules are different, for instance in scoring field goals, the attacking team need to penetrate defender $D$ area in order to make an attempt on goal, conversely in football there is no specific area in attempt on goal. The penetration of the $D$ area is one of the contributor to obtain field goals and penalty corner in field hockey, which can be proved when previous study indicate winning team were the team that most penetrated opponent area (Sofwan et al., 2012). On the other hand previous study in rugby similarly indicate the winning team has made more penetration towards opponent area to make a try (Vaz et al., 2010), moreover previous study by Tenga, Ronglan, and Bahr (2010), indicate huge potential on goal scoring and high accuracy on target when the attacker made an attempt inside the opponent score box area (penalty area) in football.

On this current study 610 times was recorded for penetration made in various ways. The most frequent were by individual run about 265 times and push about 209 times. From that figures, 589 comes by shorter passing sequence and only 21 times by longer passing sequence. This evidence established on shorter passing sequence was very effective in attacking compare to longer passing sequence. Besides that, total of penalty corners accumulated from penetration were 110 times, meanwhile 67 field goals succeed from that penetration and 5 times contributed to penalty stroke. From the penalty corners penetration 52 times contributed as goals meanwhile 3 goals succeed from penalty stroke penetration. All those figures can support the previous study that indicate more frequent penetration occurred resulted in more scored can be obtain.

Recorded in this study of all the attempts of shots on goals only $30 \%$ succeed as field goals, while others were missed or saved by the goalkeeper. Here can be explained that it is important to take any chances to score as many goals as possible when penetrate opponent's $D$ area. Besides field goals, penalty corners also important as attacking objectives in field hockey, according to De Subijana, Juarez, Mallo, and Navarro (2010) cited from previous study by Laird and Sutherland (2003); Pineiro (2008), penalty corner recorded as the most ways to obtain goals in field hockey, other study from Amjad et al. (2013), also supported that more goal chances come from penalty corners, hence study by Yusoff, Hasan, and Wilson (2008), indicate approximately from 5 penalty corners in one particular match contributed to 1 goal. As this study recorded the ratio of penalty corner goals were three trials over one goal scored.

Obviously longer passing sequence synonym with short pass, meanwhile shorter passing sequence always refer as direct play and often used long pass, however from the analysis made on this tournament most of the team likely to attack by using single player who have good quality skills in running and dribbling before made a decent pass to other player for penetrating the defender's area. This is based on the fact when individual run was the most ways to make penetration before made an attempt on goal or obtain the penalty corner, parallel with that more short passes made compared to long passes. Conversely on previous study findings by Sofwan et al. (2012) indicate most ways of D penetration was by hitting, but due to different gender ability and assessment, further study need to be done with the focus on female teams.

Basically, ball carrier is a player who running with the ball and try to attract some of the defender to seize the ball, in the meantime the receiver particularly a player without ball, make a run to create an options before receive the ball clearly and try to make an attempt on goal or find any defender's foot or other fouls to obtain penalty corners inside the 25 meters area or inside the $D$ area. The combination of quick movement and precision on passing effectively is proven to be used as attacking tactical in field hockey, as previously study on passing sequence by Tenga et al. (2010) indicate more sequence of passing to advanced towards goal make it easy for the defenders to reorganized their defensive line and immobilize the attacking team to penetrate their area. Ironically, passing is one of the basic skills that is used not only 
to deliver the ball to other player but also nowadays used to eliminate opponent to obtain as many goals as possible to become a winner at the end of the match.

Current study findings serve as guidance to any level of field hockey coaches to implement during training as to improve and enhance their team attacking ability for competition. The coaches also can used this finding to emphasize more on basic skill especially passing as well as emphasize the development on physiological ability especially speed, agility, quickness, strength, and power in order to prepare their team ready to compete in any current modern field hockey match or tournament. These findings also provide further study to be conducted in finding correlation between passing sequence and basic skills in field hockey, thus other study can be conducted on female teams to expand more findings especially in field hockey.

As a conclusion, shorter passing sequence was effectively applied as fundamentals of attacking tactical. These findings are appropriate with modern field hockey in order to obtain more field goals or collected more penalty corners as increasing the chances of scoring and become a winner at the end of the match.

\section{REFERENCES}

1. Amjad, I., Hussain, I., \& Asadullah, M. (2013). Comparison between long corners and short corners in field hockey. Rawal Medical Journal, 38(4), pp.428-431.

2. Armatas, V., Yiannakos, A., Galazoulas, C., \& Hatzimanouil, D. (2007). Goal scoring patterns over the course of a match: Analysis of women's high standard soccer matches. Physical Training, 1(1).

3. Castellano, J., Casamichana, D., \& Lago, C. (2012). The use of match statistics that discriminate between successful and unsuccessful soccer teams. Journal of Human Kinetics, 31, pp.139-147.

4. De Subijana, C. L., Juarez, D., Mallo, J., \& Navarro, E. (2010). Biomechanical analysis of the penalty-corner drag-flick of elite male and female hockey players. Sports Biomechanics, 9(2), pp.72-78.

5. International Hockey Federation. (2012). Rules of hockey, Lausane, Switzerland.

6. Hughes, M., \& Bartlett, R. (2002). The use of performance indicators in performance analysis. Journal of Sports Sciences, 20(10), pp.739-754.

7. Hughes, M., \& Franks, I. M. (2005). Analysis of passing sequences, shots and goals in soccer. Journal of Sports Sciences, 23(5), pp.509-514.

8. Lago, C., \& Martin, R. (2007). Determinants of possession of the ball in soccer. Journals of Sports Sciences, 25(9), pp.969-974.

9. Laird, P., \& Sutherland, P. (2003). Penalty corners in field hockey: A guide to success. International Journal of Performance Analysis in Sport, 3(1), pp.19-26.

10. Pineiro, R. (2008). Observation and analysis of the scoring plays in field hockey. Sevilla: Wanceulen.

11. Reep, C., \& Benjamin, B. (1968). Skill and chance in association football. Journal of the Royal Statistical Society A, 131, pp.581-585.

12. Sofwan, N., Norasrudin, S., Redzuan, P., \& Mubin, A. (2012). Distinguishing playing pattern between winning and losing field hockey team in Delhi FIH Road to London 2012 Tournament. World Academy of Science, Engineering and Technology.

13. Tenga, A., Ronglan, L. T., \& Bahr, R. (2010). Measuring the effectiveness of offensive match-play in professional soccer. European Journal of Sport Science, 10(4), pp.269-277. 
14. Vaz, L., Van Rooyen, M., \& Sampaio, J. (2010). Rugby game related statistics that discriminate between winning and losing teams in IRB and Super twelve close game. Journal of Sports Science and Medicine, 9, pp.51-55.

15. Yiannakos, A., \& Armatas, V. (2006). Evaluation of the goal scoring pattern in European Championchip in Portugal 2004. International Journal of Performance Analysis in Sport, 6(1), pp.178-188.

16. Yusoff, S., Hasan, N., \& Wilson, B. (2008). Three-dimensional biomechanical analysis of the hockey drag flick performed in competition. ISN Bulletin, National Sport Institute of Malaysia, 1(1), pp.35-43. 